\title{
Utility of two SMN1 variants to improve spinal muscular atrophy carrier diagnosis and genetic counselling
}

\author{
Laura Alías $^{1,2}$ - Sara Bernal ${ }^{1}$ - Maite Calucho ${ }^{3}$ - Elisabeth Martínez ${ }^{1}$ - Francesca March ${ }^{1}$ - Pia Gallano ${ }^{1,2}$. \\ Pablo Fuentes-Prior ${ }^{4}$ Anna Abuli, ${ }^{3,5}$. Clara Serra-Juhe ${ }^{3,5}$ - Eduardo F. Tizzano ${ }^{2,3,5}$
}

Received: 5 September 2017 / Revised: 27 April 2018 / Accepted: 9 May 2018 / Published online: 14 June 2018

(c) European Society of Human Genetics 2018

\begin{abstract}
Spinal muscular atrophy (SMA) is caused by deletions/mutations in SMN1. Most heterozygous SMA carriers have only one SMN1 copy in one of the alleles (1/0 carriers). However, a few carriers lack SMN1 in one of their chromosomes, but present two gene copies in the other. These " $2 / 0$ carriers" are undistinguishable from non-carrier individuals (1/1) with currently available methods. Previous association of SMN1 variants c.*3+80 T > G and c.*211_*212del with two SMN1 copies in cis in Ashkenazi population prompted us to analyze them in 270 Spanish individuals (SMA carriers, patients and general population). Both variants were much more frequently detected in chromosomes with 2 SMN1 copies in cis in comparison with chromosomes carrying one copy ( 17.9 vs. $0.7 \% ; p<0.001)$. In particular, one-fifth of $2 / 0$ SMA carriers harboured one or both variants compared to none of 99 non-carriers with two SMN1 copies $(p<0.001)$. The c.*211_*212del variant was also much more frequent in exon 8 of SMN2-SMN1 hybrids than in that of intact SMN1 genes (20 vs. $0.83 \%, p<0.001)$, suggesting its association with chromosomal rearrangements. Although absence of these variants does not exclude that a particular individual is a 2/0 SMA carrier, their presence is valuable to substantially increase residual risk in putative carriers, thus improving genetic counselling.
\end{abstract}

\section{Introduction}

Spinal muscular atrophy (SMA) is an autosomal recessive neuromuscular disorder with an estimated incidence of $1: 6,000$ to $1: 10,000$ live births and a carrier frequency of $1 / 35-1 / 60[1,2]$. Clinically, SMA is a continuous spectrum of phenotypes ranging from severely compromised neonates and infants to adults with minimal manifestations. Patients are classified into four main groups based on age of onset and motor milestones [3].

Electronic supplementary material The online version of this article (https://doi.org/10.1038/s41431-018-0193-4) contains supplementary material, which is available to authorised users.

Eduardo F. Tizzano

etizzano@vhebron.net

1 Servei de Genètica, Hospital de la Santa Creu i Sant Pau, Barcelona, Spain

2 CIBERER (CB06/07/0011 group), Barcelona, Spain

3 Medicine Genetics, VHIR, Barcelona, Spain

4 Molecular Bases of Disease, IIB Sant Pau, Barcelona, Spain

5 Department of Clinical and Molecular Genetics, Hospital Valle Hebron, Barcelona, Spain
Survival motor neuron 1 (SMN1) has been identified as the SMA disease-determining gene [4]. SMN2 is a highly homologous copy of $S M N 1$, which has been described as an SMA modifier $[5,6]$. In $90 \%$ of SMA cases, the molecular pathology is absence of $S M N 1$ by deletion or gene conversion and in 5\% SMN2-SMN1 hybrid genes [7-10]. The remaining SMA cases are compound heterozygous [7, 8].

Current methods of SMN1 dosage do not discriminate between 1/1 non-carriers and 2/0 carriers (individuals with two SMN1 copies in cis [2]). Familial haplotype analysis with polymorphic markers of the SMA locus is helpful to detect these $2 / 0$ carriers within blood relatives. However, diagnosis in partners of SMA carriers from the general population with two SMN1 copies is challenging [2].

Two SMN1 variants have been recently associated to chromosomes carrying two SMN1 copies in cis in the Ashkenazi Jewish population [11]. These variants, c.*3+ $80 \mathrm{~T}>\mathrm{G}$ corresponding to g.27134 $\mathrm{T}>\mathrm{G}$ in intron 7 and c.*211_*212del corresponding to g.27706_27707delAT in exon 8 of the SMN1 gene, had been previously described by Luo and co-workers [11] according to the first nucleotide of the gene, 5000 bases off from the NG_008691.1 reference sequence. Here, we tested these variants in a large set of Spanish individuals to confirm their utility to improve identification of 2/0 SMA carriers. 
Table 1 Screening for the presence/absence of variants c. $* 3+80 \mathrm{~T}>\mathrm{G}$ and c. $* 211_{-} * 212 \mathrm{del}$ in 270 Spanish individuals

\begin{tabular}{|c|c|c|c|c|c|c|c|c|}
\hline & \multicolumn{2}{|c|}{ SMA patients } & \multicolumn{3}{|c|}{ SMA carriers } & \multicolumn{3}{|c|}{ Normal individuals } \\
\hline & Del/Del & Hybrid & $1 / 0$ carriers & $2 / 0$ carriers & $3 / 0$ carriers & $2 S M N 1$ copies & $3 S M N 1$ copies & 4 SMN1 copies \\
\hline c. $* 3+80 \mathrm{~T}>\mathrm{G}$ & 0 & 0 & $1(2.4 \%)^{\mathrm{a}}$ & $7(21.8 \%)$ & 0 & 0 & $11(18.9 \%)^{\mathrm{b}}$ & 0 \\
\hline c. $* 211 \_* 212 \mathrm{del}$ & 0 & $4(20 \%)$ & $1(2.4 \%)^{\mathrm{a}}$ & $6(18.7 \%)^{\mathrm{c}}$ & 0 & 0 & $11(18.9 \%)^{\mathrm{d}}$ & 0 \\
\hline Total Ind. studied $(n=270)$ & 16 & 20 & 41 & 32 & 1 & 99 & 58 & $3^{\mathrm{e}}$ \\
\hline
\end{tabular}

${ }^{a}$ Correspond to the same individual (mother of a SMA patient with homozygous deletion of the SMN1 gene and without hybrid genes)

${ }^{\mathrm{b}}$ Ten of them presented the two variants, and other individual carried only the c.*3+80 T $>\mathrm{G}$ variant in intron 7

${ }^{c}$ All six individuals have the two variants

${ }^{\mathrm{d}}$ Ten of them presented the two variants, and other individual carried only variant c. $* 211$ - 212 del in exon 8

${ }^{\mathrm{e}}$ Note that the variants under study were not present in none of the three individuals carrying four SMN1 copies

A

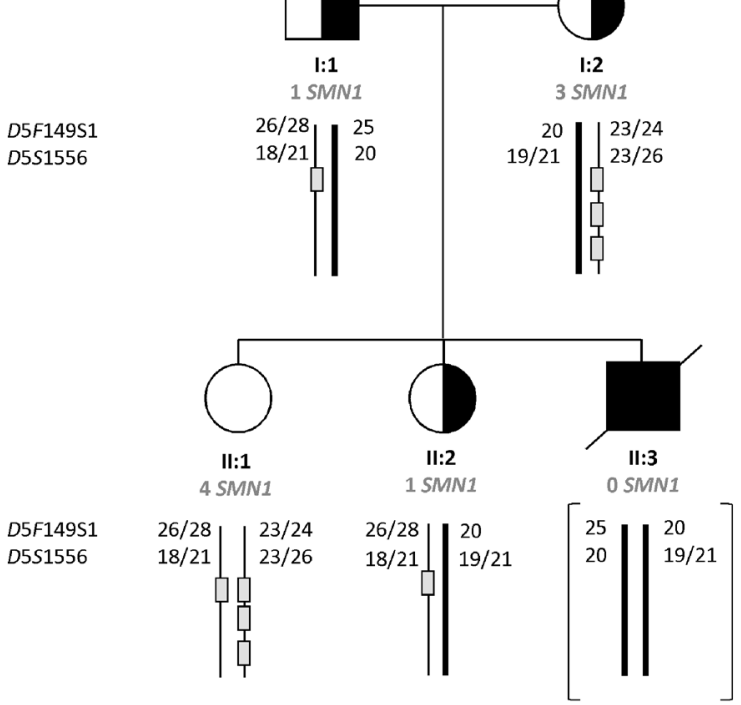

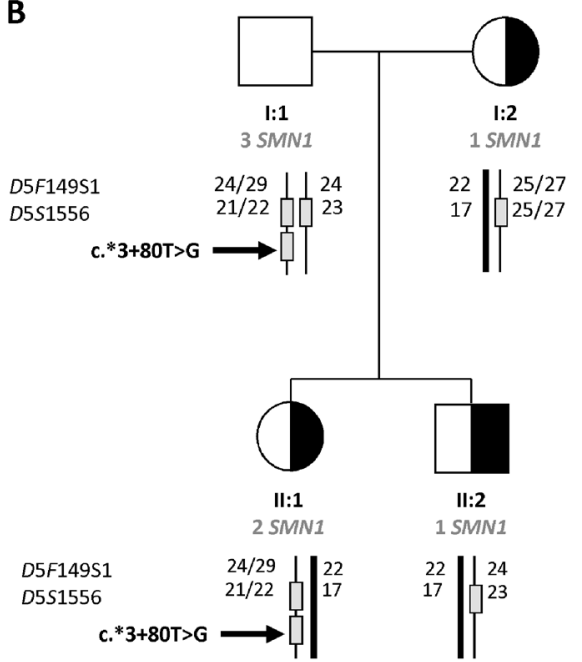

B - 
(http://databases.lovd.nl/shared/genes/SMN1) with patients' IDs 00150115, 00150116, 00150120-00150129, and 00150239-00150250. $X^{2}$ statistics were calculated with SPSS package, and $p$ values $<0.05$ were considered significant.

\section{Results}

Our major results are summarised in Table 1. In general, the studied variants were almost completely absent from chromosomes with a single SMN1 copy $(1 / 297 ; 0.33 \%)$, but they were frequently detected in those carrying two copies of the gene $(18 / 96 ; 18.75 \%)(p<0.001)$.

Carriers. One classical $1 / 0$ carrier presented the variants $(1 / 41 ; 2.4 \%)$ whereas 7 of the 32 2/0 carriers were positive $(7 / 32 ; 21.8 \%)$. Interestingly, the $3 / 0$ carrier was negative for both variants (Fig. 1a). All individuals with the variants were unrelated and shared a 20-repeat allele for marker $D 5 S 1556$. Further, five of them, who were from the Canary Islands, showed a 24-repeat allele for marker $D 5 F 149 \mathrm{~S} 1$ (Supplementary Figure 1).

Non-carriers. All $1 / 1$ individuals $(n=99)$ were negative for the studied variants, whereas 11 out of 58 subjects with three SMN1 copies $(2 / 1)$ were positive $(19.3 \% ; p<0.001)$. Four individuals in this last subgroup had also homozygous absence of SMN2 genes, three of whom showed the variants. A comparison between $2 / 0$ carriers vs. 1/1 controls was also significant $(p<0.001)$ for the presence of the variants.

SMA patients. None of the variants were found in 16 SMA patients who lack SMN1. However, four out of 20 SMA patients with SMN2-SMN1 hybrid genes presented the c.*211_*212del variant (20\%) (Supplementary Figure 2).

\section{Discussion}

To improve genetic counselling for carriers of SMA, we aimed to validate in the Spanish population two variants of the SMN1 gene, c. $* 3+80 \mathrm{~T}>\mathrm{G}$ and c.*211_*212del, which had been previously associated to chromosomes with two SMN1 copies in cis in the Ashkenazi population [11].

Our results confirm that these variants are only present in $S M N 1$. First, we did not detect them in patients with total absence of the SMN1 gene. Second, in subjects with SMN2SMN1 hybrids we detected only the c.*211_*212del variant, which corresponds to the SMN1 half of the hybrid. Finally, we identified both variants in three individuals with three SMN1 copies each, but who lack the SMN2 gene.

We have also corroborated the utility of both variants for genetic testing of SMA carriers, as they are much more frequent in chromosomes with two SMN1 copies in cis $(p<0.001)$, and are also present in almost $20 \%$ of cases with three SMN1 copies (Table 1).
We report for the first time a 3/0 SMA carrier, identified in the context of a SMA family study but without the variants (Fig. 1a). Individuals with 3 SMN1 copies are usually thought to harbour two copies in one chromosome and one in the other (2/1). Our finding emphasizes the complexity of the SMA region and points to possible pitfalls in interpreting the results of non-carriers. Genetic counselling in these cases should be carefully evaluated in the context of haplotype results [13].

Chromosomes with two SMN1 copies are more frequent in the African population [2, 16] and, concomitantly, they present both SMN1 variants at higher frequencies [11]. In our cohort, six of seven cases with the variants were from the Canary Islands, a region with African genetic influence due to the territorial proximity. Most of these cases share a common haplotype (Supplementary Figure 1).

Among non-carriers, the variants were absent in $1 / 1$ individuals but present in almost every fifth $(19.3 \%)$ of the $2 / 1$ individuals studied $(p<0.001)$, reinforcing their association with chromosomes harbouring 2 SMN1 copies in cis. These variants were previously reported in 116 out of 200 individuals $(58 \%)$ with three SMN1 copies [11]. Ninety of these positive cases were from African origin, likely explaining the differences with our cohort.

Given that the variants are linked to SMN1 but not SMN2 (ref 10. and this work), they are not detected in SMA patients who lack SMN1. However, $20 \%$ of patients with hybrid SMN2-SMN1 genes, who usually have intron 7 of the SMN2 gene and exon 8 from SMN1 [9, 10], present the exon 8linked c.*211_*212del variant. As expected, none of them had the intron 7-associated variant. These observations suggest that some hybrid genes originate from chromosomes with two SMN1 copies in cis. However, it is not known whether the presence of the variant makes SMN1 genes prone to rearrange in hybrid structures. It is also possible that individuals who only present the intron 7 variant may represent hybrid SMN1 intron 7 and SMN2 exon 8 structures.

In conclusion, our results indicate that $S M N 1$ variants c.*3 $+80 \mathrm{~T}>\mathrm{G}$ and c. $* 211_{-} * 212 \mathrm{del}$ are associated to chromosomes that underwent rearrangements such as those with two SMN1 copies in cis and those with hybrid SMN2-SMN1 genes (around $20 \%$ of the cases). However, absence of both variants in a subject with two SMN1 copies does not preclude the 2/0 carrier status limiting the utility of this analysis. Since most of the twocis chromosomes and hybrids do not show these variants, their study in SMA carrier testing may have a limited geographical application, assuming a higher frequency in the Ashkenazi Jews and African population due to an increased number of chromosomes with two SMN1 copies. However, the study of the variants is useful to select individuals with increased risk of being 2/0 carriers. Indeed, the presence of one or both variants notably increases the residual risk from $1 / 781$ to $\sim 1$ (Table 2). In these cases, testing of the parents of the individual [2] would be necessary to confirm his/her $2 / 0$ carrier status. 
Acknowledgements This work was supported by Grants from Fundación Privada Daniel Bravo Andreu (to EFT and PFP and supporting MC). LA is supported by CIBERER. We wish to thank the consenting individuals who made this study possible and colleagues for referral of families.

\section{Compliance with ethical standards}

Conflict of interest The authors declare that they have no conflict of interest.

\section{References}

1. Sugarman EA, Nagan N, Zhu H, et al. Pan-ethic carrier screening and prenatal diagnosis for spinal muscular atrophy: Clinical laboratory analysis of 72,400 specimens. Eur J Hum Genet. 2012;20:27-32.

2. Alías S, Barceló M, Bernal S, et al. Improving detection and genetic counseling in carriers of Spinal Muscular Atrophy with two copies of the SMN1 gene. Clin Genet. 2014;85:470-5.

3. Talbot K, Tizzano EF. The clinical landscape for SMA in a new therapeutic era. Gene Ther. 2017;24:529-33.

4. Lefebvre S, Bürglen L, Reboullet S, et al. Identification and characterization of a Spinal Muscular Atrophy-determining gene. Cell. 1995;80:155-65.

5. Prior TW, Krainer AR, Hua Y. et al: A positive modifier of spinal muscular atrophy in the SMN2 gene. Am J Hum Genet. 2009;85:408-13.

6. Calucho M, Bernal S, Alias L, et al: Correlation between SMA type and SMN2 copy number revisited: An analysis of 625 unrelated Spanish patients and a compilation of 2,834 reported cases. Neuromuscul Disord. 2018;28:208-15.

7. Wirth B. An update of the mutation spectrum of the survival motor neuron gene (SMN1) in autosomal recessive spinal muscular atrophy (SMA). Hum Mutat. 2000;15:228-37.

8. Alías L, Bernal S, Fuentes-Prior P, et al. Mutation update of Spinal Muscular Atrophy in Spain: molecular characterization of 745 unrelated patients and identification of four novel mutations in the SMN1 gene. Hum Genet. 2009;125:29-39.

9. Hahnen E, Schönling J, Rudnik-Schöneborn S, Zerres K, Wirth B. Hybrid survival motor neuron genes in patients with autosomal recessive spinal muscular atrophy: new insights into molecular mechanisms responsible for the disease. Am J Hum Genet. 1996;59:1057-65.

10. Cuscó I, Barceló MJ, del Rio E, et al. Characterization of SMN hybrid genes in Spanish SMA patients: de novo, homozygous and compound heterozygous cases. Hum Genet. 2001;108:222-9.

11. Luo M, Liu L, Peter I, et al. An Ashkenazi Jewish SMN1 haplotype specific to duplication alleles improves pan-ethnic carrier screening for spinal muscular atrophy. Genet Med. 2014;16:149-56.

12. Alías L, Bernal S, Barceló MJ, et al. Accuracy of marker analysis, quantitative real-time polymerase chain reaction, and multiple ligation-dependent probe amplification to determine SMN2 copy number in patients with Spinal Muscular Atrophy. Genet Test Mol Biomark. 2011;15:587-94.

13. Barceló MJ, Alías L, Caselles L, Robles Y, Baiget M, Tizzano EF. Two independent mutations of the SMN1 gene in the same Spinal Muscular Atrophy family branch: Lessons for carrier diagnosis. Genet Med. 2006;8:259-62.

14. den Dunnen JT, Antonarakis SE. Nomenclature for the description of human sequence variations. Hum Genet. 2001;109:121-4.

15. Bürglen L, Lefebvre S, Clermont O, et al. Structure and organization of the human survival motor neuron (SMN) gene. Genomics. 1996;32:479-82.

16. Hendrickson BC, Donohoe C, Akmaev VR, et al. Differences in SMN1 allele frequencies among ethnic groups within North America. J Med Genet. 2009;46:641-4. 